\title{
, neu \\ Molecular Characterization and Pathogenicity of a Novel Soybean-Infecting Monopartite Geminivirus in China
}

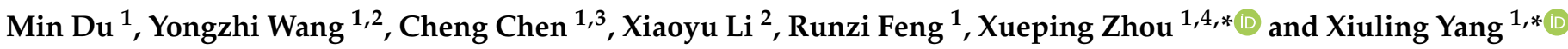 \\ 1 State Key Laboratory for Biology of Plant Diseases and Insect Pests, Institute of Plant Protection, \\ Chinese Academy of Agricultural Sciences, Beijing 100193, China; dumincaas@163.com (M.D.); \\ yzwang@126.com (Y.W.); chenfungi@126.com (C.C.); runzifeng1997@163.com (R.F.) \\ 2 Key Laboratory of Integrated Pest Management on Crops in Northeast, Ministry of Agriculture, \\ Jilin Academy of Agricultural Sciences, Changchun 130033, China; lxyzsx@163.com \\ 3 Key Laboratory of Integrated Pest Management on Crops in Southwest, Institute of Plant Protection, \\ Ministry of Agriculture, Sichuan Academy of Agricultural Sciences, Chengdu 610066, China \\ 4 State Key Laboratory of Rice Biology, Institute of Biotechnology, Zhejiang University, Hangzhou 310058, China \\ * Correspondence: zzhou@zju.edu.cn (X.Z.); xlyang@ippcaas.cn (X.Y.)
}

check for updates

Citation: Du, M.; Wang, Y.; Chen, C.; Li, X.; Feng, R.; Zhou, X.; Yang, X. Molecular Characterization and Pathogenicity of a Novel Soybean-Infecting Monopartite Geminivirus in China. Viruses 2022, 14, 341. https://doi.org/10.3390/ v14020341

Academic Editor:

Gian Paolo Accotto

Received: 4 January 2022

Accepted: 6 February 2022

Published: 8 February 2022

Publisher's Note: MDPI stays neutral with regard to jurisdictional claims in published maps and institutional affiliations.

Copyright: (C) 2022 by the authors. Licensee MDPI, Basel, Switzerland. This article is an open access article distributed under the terms and conditions of the Creative Commons Attribution (CC BY) license (https:// creativecommons.org/licenses/by/ $4.0 /)$.

\begin{abstract}
Soybean is a major legume crop that plays an important role in food production, industrial production, and animal husbandry. Here, we characterize a novel soybean-infecting monopartite geminivirus identified in China. Analysis of the contigs de novo assembled from sequenced small interfering RNAs, followed by PCR, cloning, and sequencing, the complete viral genome was determined to be 2782 nucleotides. The genome contains the conserved nonanucleotide sequence, TAATATTAC and other sequence features typical of the family Geminiviridae, and encodes two and four open reading frames in the virion-sense and the complementary-sense strands, respectively. Genome-wide pairwise identity analysis revealed that the novel virus shares less than $65.6 \%$ identity with previously characterized geminiviruses. Phylogenetic and recombination analysis indicated that this virus was placed in a unique taxon within the family Geminiviridae and potentially arose from recombination. An infectious clone of this virus was further constructed and its infectivity was tested in different species of plants. Successful infection and characteristic symptoms were observed in Glycine max, Nicotiana benthamiana, N. tabacum, N. glutinosa, and N. tabacum cv. Samsun plants. Taken together, this virus represents a member of an unclassified genus of the family Geminiviridae, for which the name soybean yellow leaf curl virus is proposed.
\end{abstract}

Keywords: geminivirus; soybean; recombination; ssDNA virus

\section{Introduction}

Geminiviridae is a family of circular single-stranded DNA (ssDNA) viruses that constitute the largest family of known plant-infecting viruses and have caused many devastating diseases in a wide range of crops, vegetables, and ornamental plants worldwide [1,2]. With the development of a high-throughput sequencing-based virus discovery technique, an increasing number of geminivirus genome sequences have been available in the last couple of years. Novel highly divergent geminiviruses, such as tomato apical leaf curl virus, mulberry mosaic dwarf-associated virus, apple geminivirus, and passion fruit chlorotic mottle virus, have been identified from both economically relevant crops and woody perennials [3-6]. Currently, geminiviruses encompass 520 species (http:/ / talk.ictvonline.org/taxonomy/, accessed on 10 November 2021) and are classified into 14 genera based on genome structure, insect vector, host range, and genome-wide pairwise sequence identity (Becurtovirus, Begomovirus, Capulavirus, Curtovirus, Eragrovirus, Grablovirus, Mastrevirus, Topocuvirus, Turncurtovirus, Maldovirus, Opunvirus, Citlodavirus, Topilevirus, Mulcrilevirus) [7,8]. Such a high number of species can be attributed to many factors, including the evolution of variants via 
mutation, recombination, and pseudo-recombination, acquisition of new components and satellites, high prevalence of insect vectors, global climate changes, and human activities [9].

The great majority of geminiviruses have a monopartite ssDNA genome that is encapsidated into twinned icosahedral particles. Their genomic components vary between 2.5 and $3.2 \mathrm{~kb}$ in length, and encode four to seven proteins through bidirectional transcription and partially overlapping open reading frames (ORFs). Members of the largest genus, Begomovirus, have either monopartite or bipartite ssDNA component(s). The replicationassociated protein (Rep) and the capsid protein (CP), encoded by the C1 and V1 ORF, are the only two proteins that are conserved across all geminiviruses. Three other proteins, the transactivation protein (TrAP) encoded by $\mathrm{C} 2$, replication enhancer protein (REn) encoded by $\mathrm{C} 3$, and the $\mathrm{C} 4$ protein, are positionally conserved across the genera Begomovirus, Curtovirus, Topocuvirus, Turncurtovirus, Maldovirus, and Opunvirus, but the functions of these proteins vary and possibly are diverse from different lineages of geminiviruses.

Soybean (Glycine max) is a major legume crop that is known for its rich protein and oil content. It plays an important role in food production, industrial production, and animal husbandry. Similar to many agriculturally important crops, soybean plants are constantly attacked by both abiotic and biotic stresses and a handful of soybean viral diseases have been documented [10]. Viruses such as soybean mosaic virus, soybean dwarf virus, bean pod mottle virus, cowpea mild mottle virus, and alfalfa mosaic virus are the major reported soybean-infecting viruses that have posed a serious economic threat to soybean production, typically causing 10-30\% yield losses under natural field conditions. Fifty percent to eighty percent yield losses were also reported for severe outbreaks [11]. In this study, we conducted a high-throughput sequencing of small RNAs isolated from diseased soybean leaf samples, collected from Jilin province of China. We identified a novel highly divergent geminivirus and demonstrated the infectivity of the new virus in its natural host and experimental hosts. The name soybean yellow leaf curl virus (SbYLCV) is proposed and could be considered as a type member of a new putative genus of the Geminiviridae family.

\section{Materials and Methods}

\subsection{Plant Materials and Growth Conditions}

Soybean plants showing yellowing and dwarf symptoms were collected from Jilin province of China in 2018. Glycine max cv. Williams 82, Nicotiana benthamiana, N. tabacum, N. tabacum cv. Samsun NN, N. tabacum cv. Samsun. nn, N. glutinosa, and Solanum lycopersicum cv. Moneymaker plants used for agrobacterium-mediated inoculation were grown in an insect-free growth room at $25^{\circ} \mathrm{C}$ with a $16: 8 \mathrm{~h}$ (light/dark) photoperiod.

\subsection{Small RNA-Based Deep Sequencing}

Total RNA was extracted from $1.0 \mu \mathrm{g}$ of symptomatic soybean plant leaves using TRIzol reagent following the manufacturer's protocols (Invitrogen, Carlsbad, CA, USA). Small RNAs (sRNAs) were purified from the extracted total RNA using Small RNA Sample Pre Kit (Illumina, San Diego, CA, USA), which were sequentially ligated with $3^{\prime}$ and $5^{\prime}$ adapters, reverse transcribed to complementary DNA (cDNA), and PCR amplified as described [12]. The resulting sRNA cDNA library was subjected to deep sequencing on an Illumina HiSeq ${ }^{\mathrm{TM}} 2000$ platform (Novogene, Beijing, China). After the removal of adapter sequences and low-quality reads, high-quality clean reads were assembled using the Velvet program [13]. The assembled contigs were subsequently screened for candidate virus(es) by BLASTn on the GenBank Virus Reference Sequence Database.

\subsection{DNA Extraction and Amplification of the Full-Length Viral Genome}

Total DNA was extracted from plant leaves using a cetyltrimethyl ammonium bromide (CTAB)-based extraction procedure, as described [14]. Genomic DNA was amplified by rolling circle amplification (RCA) with Templiphi ${ }^{\mathrm{TM}}$ Kit (GE Healthcare, Sugar Notch, PA, USA) following the manufacturer's protocols. RCA products were diluted five-fold 
and used for polymerase chain reaction (PCR) amplification using a pair of adjacent primers, Virus-F1/Virus-R1 (Table S1), designed based on assembled viral contigs identified by BLAST analysis. PCR was conducted with the TransStart FastPfu DNA polymerase (TransGen Biotech, Beijing, China) following the manufacturer's instructions. The PCR program was set as follows: initial denaturation at $95^{\circ} \mathrm{C}$ for $2 \mathrm{~min}$; 35 cycles of denaturation at $95{ }^{\circ} \mathrm{C}$ for $20 \mathrm{~s}$, annealing at $55^{\circ} \mathrm{C}$ for $20 \mathrm{~s}$, and amplification at $72{ }^{\circ} \mathrm{C}$ for $2 \mathrm{~min}$; and a final extension at $72{ }^{\circ} \mathrm{C}$ for $5 \mathrm{~min}$. The expected 2.8-kb PCR product was confirmed by electrophoresis and gel-purified using the E.Z.N.A. Gel Extraction Kit (Omega, Norcross, GA, USA). The purified fragments were cloned into the pEASY-T-Blunt vector (TransGen) and transformed into competent Escherichia coli $\mathrm{DH} 5 \alpha$ cells. The recombinant clones were screened and sequenced with universal primer pairs, M13F/M13R, and walking primers via Sanger sequencing (TsingKe Biotech Co., Beijing, China). Based on the full-length genome sequence, the other pair of adjacent primers, Virus-F2/Virus-R2 (Table S1), were used to amplify the full-length fragment via a similar strategy.

\subsection{Genome Characterization}

Sequences were edited and assembled using the Lasegene 7 Software (DNASTAR Inc., Madison, WI, USA). Open reading frames (ORFs) encoded by the complete viral genome were predicted using ORF Finder (http:/ / www.ncbi.nlm.nih.gov/gorf/gorf.html, accessed on 27 November 2019). The reconstructed genome sequences were subjected to the BLASTn algorithm (https:/ / blast.ncbi.nlm.nih.gov / Blast.cgi, accessed on 27 November 2019) to identify which of the sequences were most related to.

\subsection{Pairwise Distances, Phylogenetic, and Recombination Analysis}

The representative complete genome sequences of the 14 genera of the family Geminiviridae were retrieved from the GenBank database and used for delimiting species boundaries. The amino acid sequences of inferred capsid protein $(\mathrm{CP})$ and replication-associated protein (Rep) were selected from the complete viral genomes. Pairwise nucleotide sequence comparisons for the full-length genome and amino acid sequence comparisons for Rep and $\mathrm{CP}$ were conducted using the Sequence Demarcation Tool (SDT) v1.2 [15]. Alignment of the nucleotide or amino acid sequences of target sequences was performed using Clustal $\mathrm{W}$ and the neighbor-joining phylogenetic trees were constructed with 1000 bootstrap replicates using the MEGA software version 5.0 [16]. Recombination analysis was carried out using the recombination detection program RDP4 [17]. Sequences were aligned in Muscle in MEGA5 and exported to the RDP4 program to test potential recombination events, using more than one method for authenticity.

\subsection{Infectivity Assays}

To generate the infectious clone of SbYLCV, two-tandem repeats of SbYLCV were cloned into the pBinPLUS vector using a one-step assembly strategy [18]. In brief, two pairs of primers were designed based on the complete sequence of SbYLCV and the pBinPLUS vector, with $15 \mathrm{bp}$ overlapping nucleotides at fragment ends. Primer sets, SbYLCV-SalI-1F/SbYLCV-1R and SbYLCV-2F/SbYLCV-EcoRI-2R (Table S1) were used to amplify individual full-length fragments of SbYLCV. PCR reactions were conducted with TransStart FastPfu high-fidelity DNA polymerase (TransGen) with DNA of the Jilin isolate as a template. The pBinPLUS vector was digested with FastDigest restriction enzymes, SalI and EcoRI. PCR products and the linearized pBinPLUS vector were gel-purified with E.Z.N.A Gel Extraction Kit (Omega Bio-Tek, Norcross, GA, USA). The two overlapping full-length fragments of SbYLCV were seamlessly assembled to the linearized pBinPLUS vector using the In-Fusion ${ }^{\circledR}$ HD Cloning Kit according to the manufacturer's instructions (Takara, Tokyo, Japan). The positive clones were screened by colony PCR and plasmids were extracted and digested with the restriction enzyme, EcoRI to verify the correct insertion of two tandem repeats. Three independent clones were further sequenced to ensure that no 
mutations were introduced. The resultant recombinant clone, pBinPLUS-SbYLCV 2A was transformed into Agrobacterium tumefaciens EHA105 by electroporation.

\subsection{Agroinoculation of Plants}

Assays regarding the infectivity of the infectious clone of SbYLCV in tested plants were conducted, as previously described [19]. A. tumefaciens clones carrying the two tandem repeats of SbYLCV were cultured in LB broth with kanamycin $(50 \mu \mathrm{g} / \mathrm{mL})$ and rifampicin $(50 \mu \mathrm{g} / \mathrm{mL})$ overnight at $28{ }^{\circ} \mathrm{C}$. After centrifugation at $4000 \times g$ for $10 \mathrm{~min}$, the pelleted agrobacteria cells were resuspended to an optical density $\mathrm{OD}_{600}$ of 1.0 with infiltration buffer ( $10 \mathrm{mM} \mathrm{MgCl} 2,10 \mathrm{mM}$ MES ( $\mathrm{pH}$ 5.8) and $100 \mu \mathrm{M}$ acetosyringone), and incubated at room temperature for $2-3 \mathrm{~h}$ before infiltration. To test the infectivity of the infectious clone of SbYLCV in solanaceous plants, plants at the 4-6 leaf stage were used for agroinoculation, as described [19]. For agroinoculation of N. benthamiana, N. tabacum, and N. glutinosa, resuspended agrobacteria cells were infiltrated into the abaxial surface of plant leaves using a $1 \mathrm{~mL}$ needleless syringe. For agroinoculation of tomato plants, the agrobacteria suspension was injected into the stems and abaxial leaves using a $1 \mathrm{~mL}$ fine syringe. For agroinoculation of soybean plants, cotyledon knots of two-day-old sprouted soybean seeds were injected with agrobacteria suspension using a $1 \mathrm{~mL}$ fine syringe. In all the experiments, plants inoculated with $A$. tumefaciens containing the empty vector, pBinPLUS were used as negative controls. Inoculated plants were placed in an insect-free greenhouse and visualized daily for symptom development. Photos were taken with a Canon 400D digital camera.

\subsection{Southern Blot Assay}

Nucleic acids were isolated from young leaves of inoculated plants at 30 days postinoculation. Samples of total genomic DNA $(20 \mu \mathrm{g})$ were electrophoresed in $1.5 \%$ agarose gel and stained with ethidium bromide to provide a loading control. After denaturation and neutralization, total DNA was transferred to Hybond $\mathrm{N}^{+}$nylon membranes (GE Healthcare) by capillary transfer. Membranes were hybridized at $50{ }^{\circ} \mathrm{C}$ to digoxigenin-labeled probes specific to SbYLCV. After washing, the hybridization signals were detected using the anti-digoxigenin AP chemiluminescent substrate CSPD following the manufacturer's instructions (Roche Diagnostics, Rotkreuz, Switzerland). The results were visualized with a chemiluminescence detection system (Tianneng, Shanghai, China).

\section{Results}

\subsection{Discovery of a Novel Soybean-Infecting Monopartite Geminivirus}

To identify possible viruses in the collected soybean samples, total RNA was extracted from symptomatic leaves and subjected to deep sequencing using the Illumina Hiseq2000 platform. A total of 20,380,853 clean reads were obtained after filtering the $20,914,978$ raw reads by trimming low-quality reads, $5^{\prime}$ - and $3^{\prime}$-adapter sequences, and poly $\mathrm{A} / \mathrm{T} / \mathrm{C} / \mathrm{G}$ reads. De novo assembly of the small RNA reads using the Velvet program generated 999 long contigs. BLASTn searches of the long contigs against the GenBank nucleotide database revealed that the longest contig (347 nucleotides) was closely related to begomovirus $\mathrm{C} 1$, sharing the highest nucleotide identity $(85 \%)$ with ageratum yellow vein virus isolate AFSP8d (accession number JN809826.1). Considering the circular nature of the geminivirus genome, adjacent primers Virus-F1/Virus-R1 were designed based on the longest contig to amplify the putative full-length geminivirus-like genome. Two symptomatic soybean leaf samples and one asymptomatic soybean sample were used for the detection of the candidate virus. Amplicons of approximately $3.0 \mathrm{~kb}$ in length were amplified from the two symptomatic soybean leaf samples, but not from the asymptomatic soybean leaves. Cloning and sequencing of the amplicons generated inserts of 2782 nucleotides (nts) in size. To reconfirm the circular characteristic of the soybean-infecting DNA molecule, another pair of adjacent primers, Virus-F2/Virus-R2, were designed based on the $2782 \mathrm{bp}$ sequence. A band of about $3 \mathrm{~kb}$ was also successfully amplified from the symptomatic soybean leaves. 
Cloning and sequencing of the fragment revealed that the amplified $2782 \mathrm{bp}$ sequence was identical to the sequence obtained by Virus-F1/Virus-R1, suggesting that the amplified $2782 \mathrm{bp}$ sequence represents the full genome of a circular DNA virus. The whole genome sequence has been deposited in Genbank (accession number OL404964). BLASTn analysis, using the $2782 \mathrm{bp}$ sequence as a query, showed that the region from 1615 to $2782 \mathrm{nt}$ has a top similarity (83\%) with tomato leaf curl Java virus-[Ageratum] (ToLCJV) (accession number AB162141.1), and the other region of about 1600 nt has no significant similarity with known sequences. Pairwise identity of the $2782 \mathrm{bp}$ sequence with the complete genome sequence of ToLCJV revealed that they share $65.6 \%$ genome-wide pairwise identity, supporting the likelihood that the circular DNA isolated from the symptomatic soybean leaves represents a previously uncharacterized geminivirus.

To determine whether the identified soybean-infecting geminivirus is associated with a putative DNA-B component, an alphasatellite, or a betasatellite, the DNA sample obtained from the same diseased soybean plant was used as a template and PCR was performed using degenerate primer pairs, CR01/CR02 [20], UNA101/UNA102 [21], and $\beta 01 / \beta 02$ [22], respectively. Consistent with the deep sequencing data, no amplified product was detected with any of the primer sets (data not shown). Taken together, the results suggest that the soybean-infecting DNA molecular is a monopartite geminivirus, for which the name soybean yellow leaf curl virus (SbYLCV) is tentatively proposed.

\subsection{Genome Organization of SbYLCV}

By using the ORF finder (https:/ / www.ncbi.nlm.nih.gov/orffinder/, accessed on 27 November 2019), six recognizable ORFs-encoding proteins composed by $>96$ amino acids were identified, considering both strands of the SbYLCV genome. The arrangement of the six ORFs within the SbYLCV genome resembles those described for monopartite begomoviruses, topocuviruses, turncurtoviruses, maldoviruses, and opunviruses, with two ORFs (V1 and V2) on the virion strand and four $(\mathrm{C} 1, \mathrm{C} 2, \mathrm{C} 3$, and C4) on the complementary strand (Figure 1). The virion and complementary strands of SbYLCV are separated by an intergenic region (IR) of 285 nts, which shares a conserved nonanucleotide motif, TAATATTAC, with most geminiviruses (Figure 1 and Table 1).

Table 1. ORFs and encoded proteins in the genome of soybean yellow leaf curl virus.

\begin{tabular}{|c|c|c|c|c|c|c|c|}
\hline ORF & $\begin{array}{l}\text { Nucleotide } \\
\text { Coordinates }\end{array}$ & Orientation & $\begin{array}{l}\text { No. Amino } \\
\text { Acids }\end{array}$ & $\begin{array}{c}M_{\mathrm{r}} \\
(\mathrm{kDa})\end{array}$ & BLASTP (E-Value) & $\begin{array}{l}\text { Predicted } \\
\text { Domains } \\
\text { (SMART) }\end{array}$ & $\begin{array}{c}\text { Amino Acid } \\
\text { Coordinates } \\
\text { of Predicted } \\
\text { Domain }\end{array}$ \\
\hline V1 & 263-1048 & Sense & 262 & 29.4 & $\begin{array}{c}\text { Coat protein of sweet } \\
\text { potato symptomless } \\
\text { virus } 1\end{array}$ & $\begin{array}{l}\text { Geminivirus coat } \\
\text { protein/nuclear } \\
\text { export factor BR1 } \\
\text { family }(1.04 \mathrm{E}-10)\end{array}$ & $5-259$ \\
\hline $\mathrm{V} 2$ & $139-450$ & Sense & 129 & 11.9 & $\begin{array}{l}\text { V2 of Juncus maritimus } \\
\text { associated virus }\end{array}$ & - & - \\
\hline $\mathrm{C} 1$ & 2637-1552 & Complementary & 362 & 41.3 & $\begin{array}{c}\text { Rep of ageratum } \\
\text { yellow vein China virus }\end{array}$ & $\begin{array}{c}\text { Geminivirus Rep } \\
\text { catalytic domain } \\
(3.04 \mathrm{E}-62) \\
\text { Geminivirus rep } \\
\text { protein central } \\
\text { domain (5.73E-35) }\end{array}$ & $\begin{array}{r}7-119 \\
126-230\end{array}$ \\
\hline $\mathrm{C} 2$ & $1649-1233$ & Complementary & 139 & 15.4 & $\begin{array}{l}\text { C2 of Juncus maritimus } \\
\text { associated virus }\end{array}$ & $\begin{array}{l}\text { Geminivirus AL2 } \\
\text { protein }(3.18 \mathrm{E}-20)\end{array}$ & $1-135$ \\
\hline C3 & 1537-1082 & Complementary & 152 & 17.6 & $\begin{array}{l}\text { C3 of Juncus maritimus } \\
\text { associated virus }\end{array}$ & $\begin{array}{c}\text { Geminivirus AL3 } \\
\text { protein } \\
(4.13 \mathrm{E}-35)\end{array}$ & $18-132$ \\
\hline $\mathrm{C} 4$ & 2480-2190 & Complementary & 97 & 10.5 & $\begin{array}{c}\text { C4 of ageratum yellow } \\
\text { vein virus }\end{array}$ & $\begin{array}{l}\text { Geminivirus C4 } \\
\text { protein }(1.72 \mathrm{E}-35)\end{array}$ & $1-84$ \\
\hline
\end{tabular}




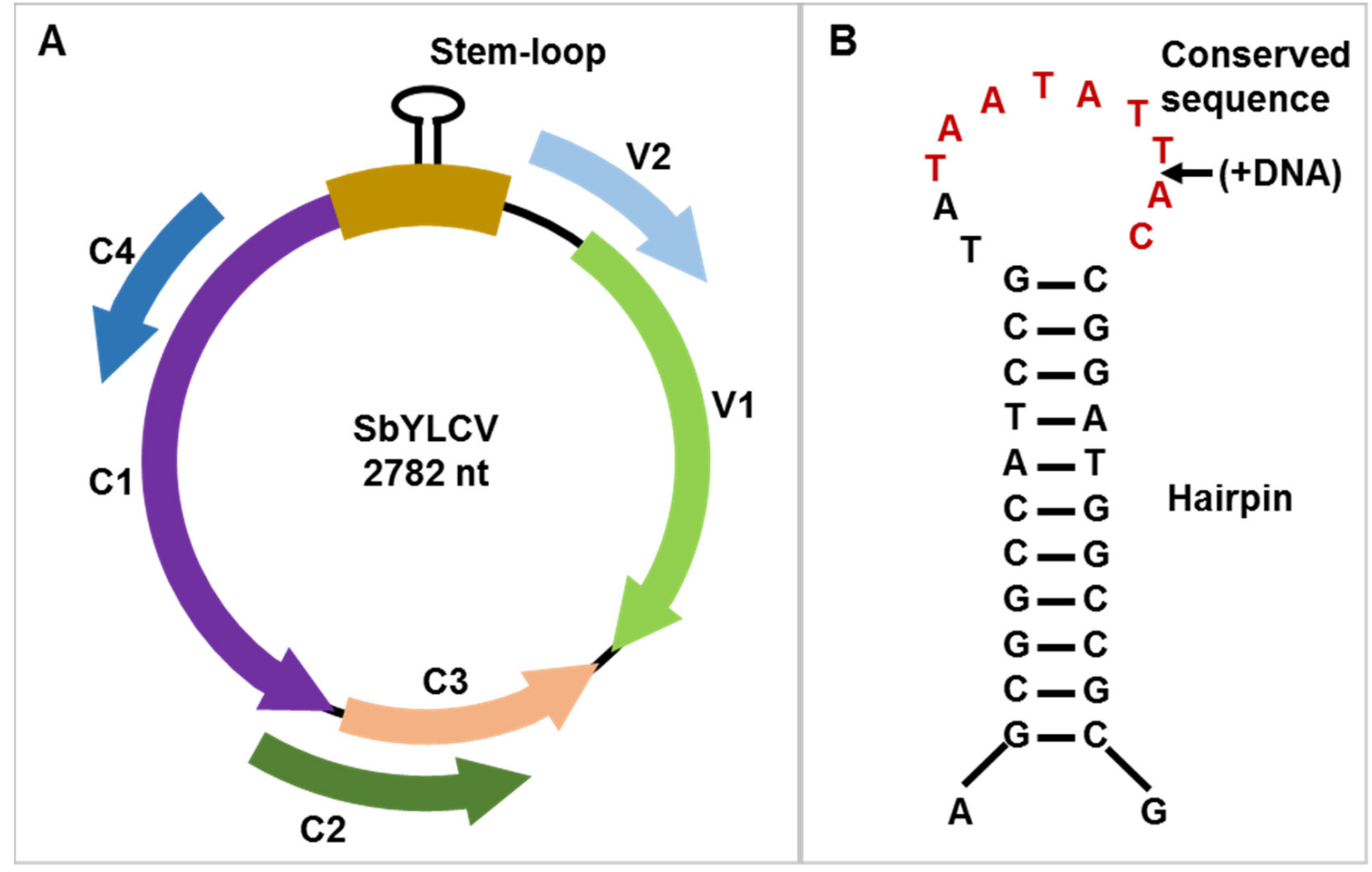

Figure 1. Genome organization of soybean yellow leaf curl virus (SbYLCV). (A) Schematic representation of SbYLCV genomic organization. Open reading frames encoded on the virion-sense $(\mathrm{V})$ strand and complementary-sense (C) strand are denoted with different colors. (B) The stem-loop structure located within the intergenic region of SbYLCV. The conserved nonanucleotide sequence is shown in red color. The putative nick site within the nonanucleotide sequences is marked with an arrow.

BLASTP searches using the amino acid sequences of the six individual ORFs of SbYLCV revealed that most of the predicted SbYLCV proteins are highly divergent from those of known geminiviruses. The predicted V1 protein of SbYLCV shares a $32.37 \%$ of amino acid sequence identity with the coat protein $(\mathrm{CP})$ of sweet potato symptomless virus 1. SMART analysis showed that the V1 protein was identified as a geminivirus CP. Although the predicted V2 protein had the highest amino acid sequence identity $(41.89 \%)$ with the V2 protein of Juncus maritimus-associated virus, SMART analyses did not recognize any conserved domains in SbYLCV V2 (Table 1). On the contrary, the predicted $\mathrm{C} 1$ protein shares a high homology $(87.72 \%)$ with the Rep protein of ageratum yellow vein China virus. Examination of the conserved domains by SMART analysis revealed that both the conserved catalytic and central domains of geminivirus Rep proteins were found in SbYLCV C1. A comparison of SbYLCV C1 to selected geminiviruses indicated that $\mathrm{SbYLCV}$ contains typical domains required for geminivirus rolling circle replication: motif I (FLTYP) is required for specific dsDNA binding, motif II (HLH) is involved in protein conformation and DNA cleavage, motif III (YI/xD/EKD) is related to DNA cleavage, and a geminivirus Rep sequence (GRS)-conserved motif is responsible for rolling circle replication initiation (Figure 2) [23-25]. The predicted C2 and C3 protein of SbYLCV shares 51.22\% and $51.26 \%$ identity with those of Juncus maritimus-associated virus. Similar to C1, the predicted SbYLCV C4 protein displays less divergence and shares the highest amino acid sequence identity $(82.29 \%)$ with that of ageratum yellow vein virus. Taking into account the nucleotide sequence divergence of the full-length genome and the amino acid sequence of the predicted proteins encoded by SbYLCV, SbYLCV identified in this study is distinct from currently classified geminiviruses and represents a novel species. 

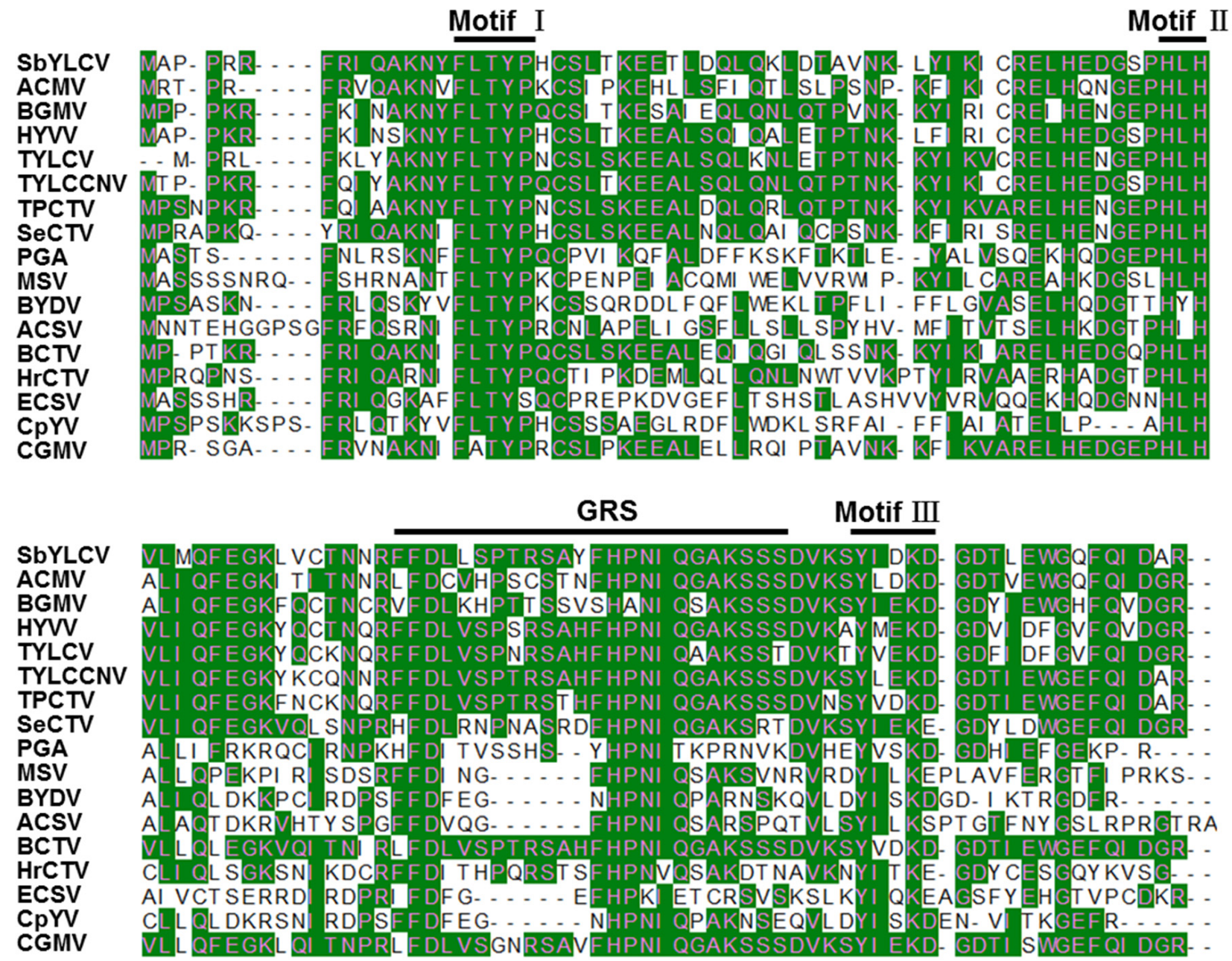

Figure 2. Alignment of the N-terminal sequences of the predicted SbYLCV Rep protein with those of selected geminiviruses. The conserved motifs I, II, III, and GRS are shown, as indicated. ACMV, African cassava mosaic virus; ACSV, Axonopus compressus streak virus; BCTV, beet curly top virus; BGMV, bean golden mosaic virus; BYDV, bean yellow dwarf virus; CGMV, cowpea golden mosaic virus; CPYV, chickpea yellows virus; ECSV, Eragrostis curvula streak virus; HrCTV, horseradish curly top virus; HYVV, honeysuckle yellow vein virus; MSV, maize streak virus; PGA, prunus geminivirus A; SeCTV, sesame curly top virus; TPCTV, tomato pseudo-curly top virus; TYLCCNV, tomato yellow leaf curl China virus; TYLCV, tomato yellow leaf curl virus.

\subsection{Phylogenetic Relationships}

To understand the evolutionary relationship of SbYLCV to other known geminiviruses, phylogenetic trees were individually constructed using the nucleotide sequences of the full-length genomes and the amino acid sequences of CP and Rep encoded by selected geminiviruses. The full-length genome-based phylogeny showed that SbYLCV does not group with geminiviruses belonging to any of the established 14 genera (Figure 3A). Pairwise distance analysis from the color-coded matrix plot revealed that SbYLCV shares 43 65.6\% genome-wide pairwise identities with other geminiviruses (Figure S1).

Similarly, phylogenetic analysis of the predicted SbYLCV CP amino acid sequences with those of representative geminiviruses revealed that SbYLCV CP forms a distinct clade and is most closely related to mastreviruses and the Eragrostis curvula streak virus (ECSV) (Figure 3B). Comparison of protein sequences revealed that the predicted CP protein of SbYLCV share $<33 \%$ amino acid sequence similarity with the CP of other geminiviruses (Figure S2). 


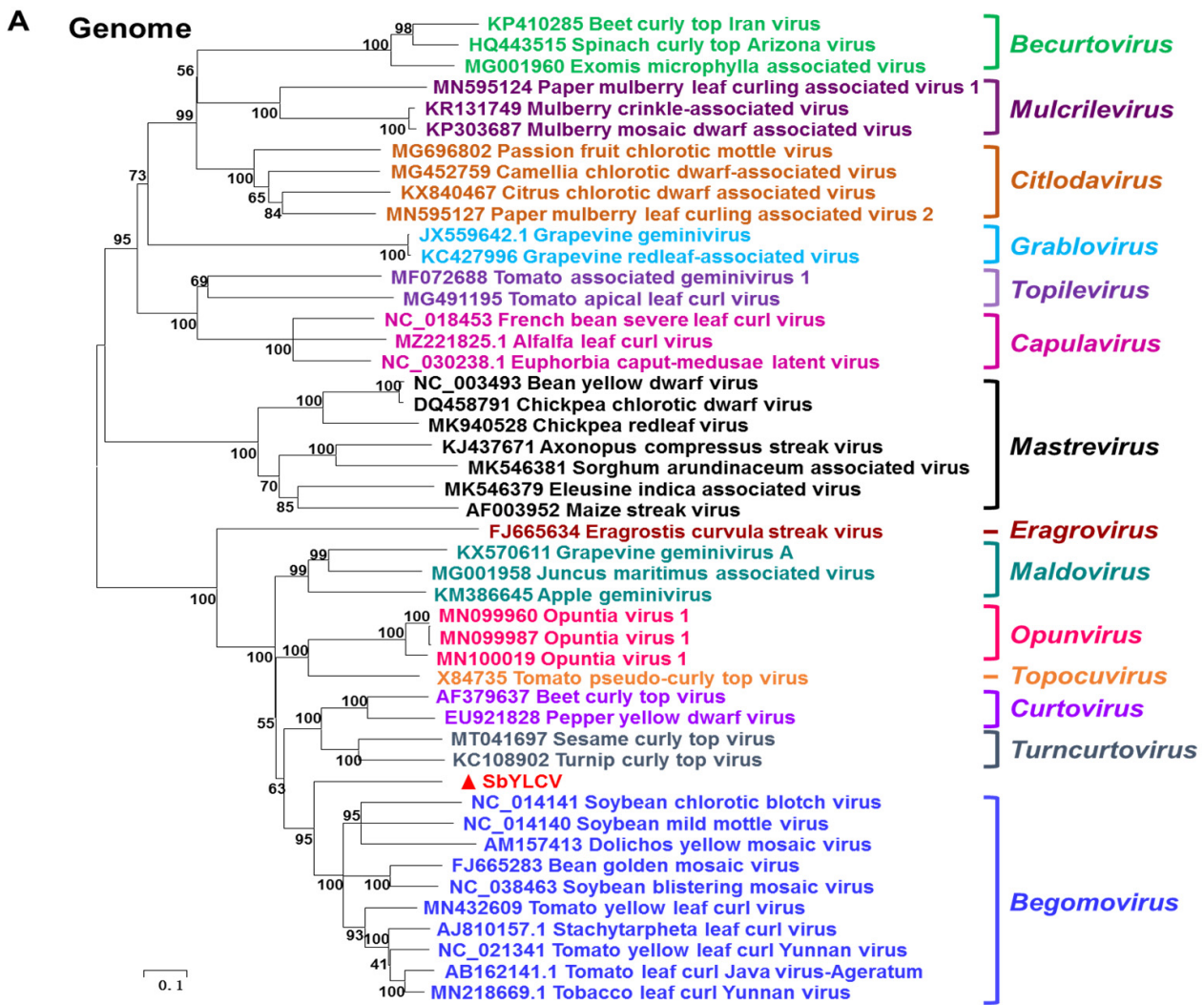

B CP

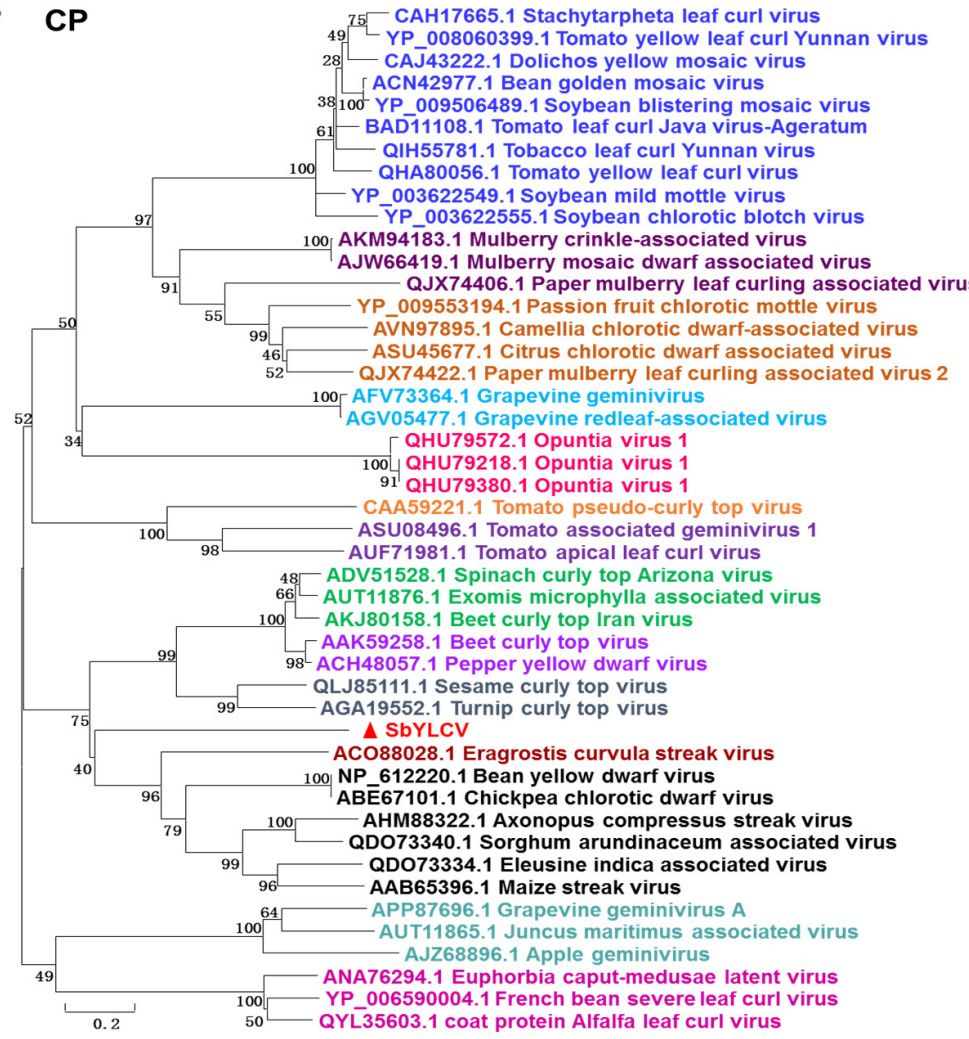

Begomovirus
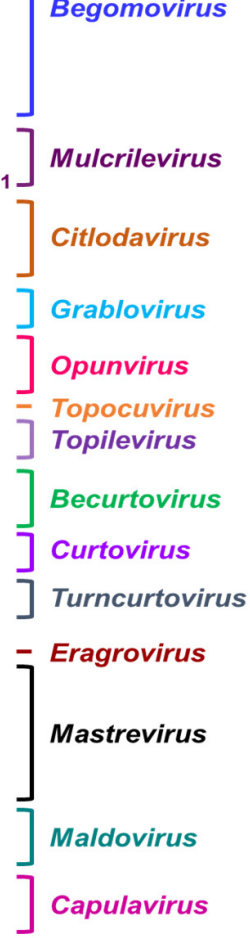

Figure 3. Phylogenetic relationships of SbYLCV and representative geminiviruses based on the nucleotide sequence of the full-length genome (A) and the amino acid sequence of the coat protein (CP) (B). The phylogenetic trees were constructed with MEGA 5.0 using the neighbor-joining method. The statistical significance of the branches was determined with a bootstrap of 1000 replicates. 
When the phylogenetic tree was generated using SbYLCV Rep amino acid sequences together with the Rep of other representative geminiviruses, it was inferred that the SbYLCV Rep sequence groups together with the Rep of begomoviruses (Figure S3), an observation that was consistent with the high sequence identities of the pairwise distance analysis (Figure S4).

\subsection{Recombination Analysis}

As recombination plays a pivotal role in driving the emergence and evolution of geminiviruses [26], the RDP software was used to examine the SbYLCV sequence for evidence of recombination. One recombination event was detected with a high degree of confidence by six recombination detection methods: RDP (average $p$ value, $2.66 \times 10^{-24}$ ), GENECONV (average $p$ value, $3.79 \times 10^{-2 \circ}$ ), Bootscan (average $p$ value, $2.79 \times 10^{-21}$ ), MaxChi (average $p$ value, $1.04 \times 10^{-17}$ ), Chimera (average $p$ value, $3.05 \times 10^{-4}$ ), and SiSscan (average $p$ value, $2.79 \times 10^{-23}$ ). The breakpoints for this predicted event ranged from nucleotides 1674 to 2760 . The sequence of stachytarpheta leaf curl virus (accession number AJ810157.1) in the analyzed dataset was implicated as the putative minor parent, and those of Dolichos yellow mosaic virus (accession number AM157413) and an unknown virus, resemble the putative major parent (Figure 4). Hence, SbYLCV is a novel virus arising from recombination.

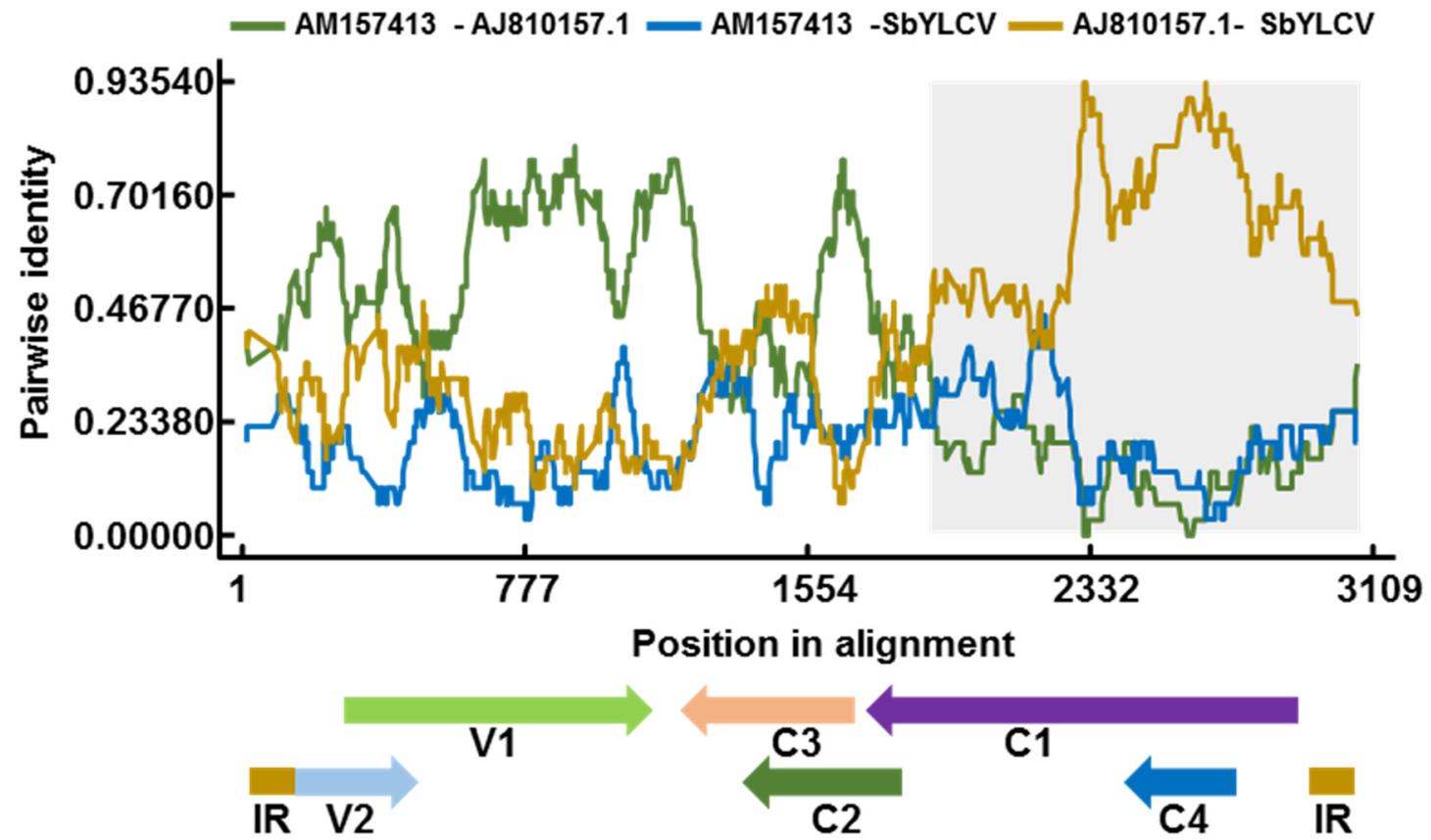

Figure 4. Recombination analysis of SbYLCV. The recombinant event was detected using the Recombination Detection Program RDP4. The brown line indicates the pairwise identity between the minor parent (Stachytarpheta leaf curl virus, AJ810157.1) and SbYLCV. The grey region represents the location of predicted breakpoints. The displayed linearized genome organization of SbYLCV shows the position in the alignment.

\subsection{Infectivity of the Infectious Clone of SbYLCV}

To test the infectivity of SbYLCV, two-tandem repeats of SbYLCV full-length fragments were cloned into the binary vector $\mathrm{pBinPLUS}$ to generate pBin-SbYLCV-2A. Infectivity tests were first performed in soybean (natural host) and $N$. benthamiana plants, the widely used model plant for studying the virus-host interaction. At 14 days post-agroinoculation (dpi), five of the 52 soybean plants inoculated with pBin-SbYLCV-2A displayed leaf curling and yellowing symptoms, which were visually different from that inoculated with the empty vector pBinPLUS (mock treatment; Figure 5A, Table 2). Leaf curling and stunting 
symptoms were also observed in all the $N$. benthamiana plants $(n=34)$ agroinfiltrated with pBin-SbYLCV-2A, but not in those agroinfiltrated with the empty vector pBinPLUS (Figure 5A, Table 2). Southern blot hybridization, using a specific probe for SbYLCV, demonstrated the systemic infection and efficient replication of SbYLCV in both soybean and $N$. benthamiana plants (Figure 5B), indicating that the developed infectious clone of SbYLCV is infectious in both soybean and N. benthamiana plants.

A

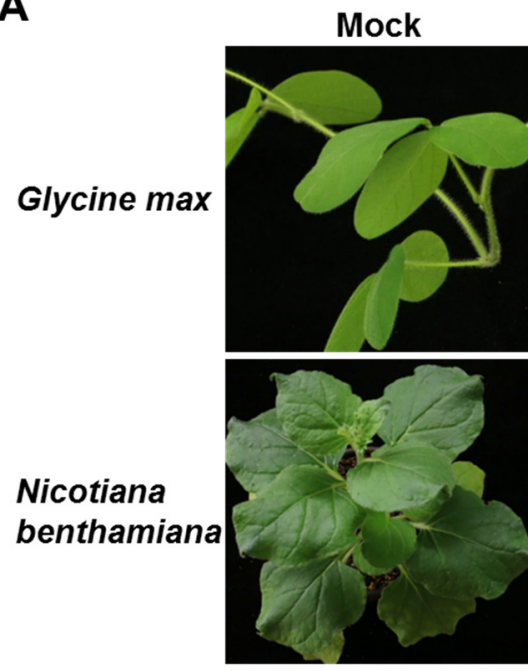

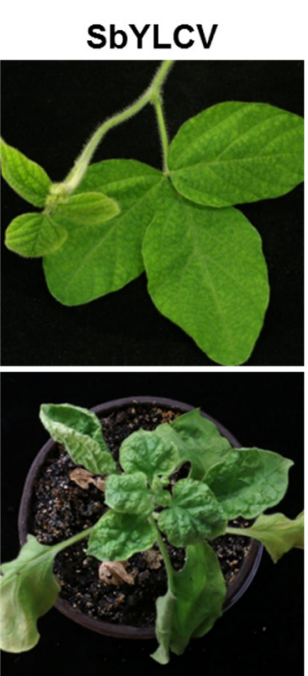

B

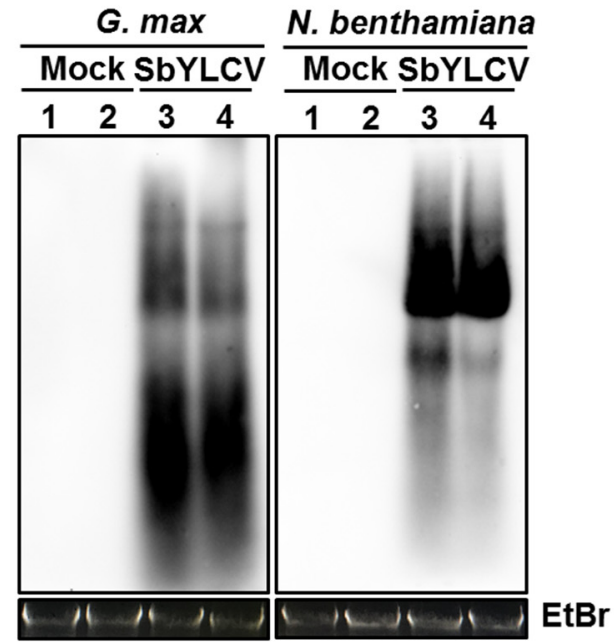

Figure 5. Systemic infection of SbYLCV in Glycine max and Nicotiana benthamiana plants. (A) Characteristic symptoms induced by SbYLCV. Plants were agroinoculated with the pBinPLUS vector (mock) or the infectious clone of SbYLCV, as indicated. Photos were taken at 30 days post-inoculation (dpi). (B) Southern blot hybridization analysis of SbYLCV DNA with a specific DIG-labelled DNA probe. Total DNA was extracted from systemic leaves of SbYLCV-infected and mock-inoculated plants, as indicated. Ethidium bromide staining of total DNA was used as loading controls.

Table 2. The infectivity analysis of soybean yellow leaf curl virus.

\begin{tabular}{|c|c|c|c|c|}
\hline Plants & $\begin{array}{l}\text { No. of Plants } \\
\text { Infiltrated }\end{array}$ & $\begin{array}{c}\text { No. of Plants } \\
\text { Systemically } \\
\text { Infected }\end{array}$ & $\begin{array}{c}\text { Infection } \\
\text { Efficiency (\%) }\end{array}$ & Symptoms \\
\hline $\begin{array}{c}\text { Nicotiana } \\
\text { benthamiana }\end{array}$ & 34 & 34 & $100 \%$ & Leaf curling \\
\hline Glycine max & 52 & 5 & $9.6 \%$ & $\begin{array}{c}\text { Leaf curling, } \\
\text { yellowing }\end{array}$ \\
\hline $\begin{array}{c}\text { Solanum } \\
\text { lycopersicum }\end{array}$ & 15 & 0 & 0 & NA \\
\hline $\begin{array}{l}\text { Nicotiana } \\
\text { tabacum }\end{array}$ & 16 & 13 & $81.3 \%$ & Leaf curling \\
\hline $\begin{array}{c}\text { Nicotiana } \\
\text { tabacum } \\
\text { cv. Samsun NN }\end{array}$ & 10 & 7 & $70 \%$ & Leaf curling \\
\hline $\begin{array}{c}\text { Nicotiana } \\
\text { tabacum } \\
\text { cv. Samsun nn }\end{array}$ & 10 & 3 & $33 \%$ & Leaf curling \\
\hline $\begin{array}{l}\text { Nicotiana } \\
\text { glutinosa }\end{array}$ & 10 & 2 & $20 \%$ & Leaf curling \\
\hline
\end{tabular}

As many geminiviruses can infect Solanaceae crops, the infectivity of SbYLCV was further assessed on four other plant species of the family Solanaceae. Agroinoculated plants were closely monitored for symptom development. At 21 dpi, a typical leaf curling symptom was visible in the systemic leaves of N. tabacum, N. tabacum cv. Samsun, and 
N. glutinosa plants agroinfiltrated with pBin-SbYLCV-2A (Figure 6A, Table 2). Detection of viral DNA using Southern blot hybridization inferred the accumulation of viral DNA in these symptomatic leaves (Figure 6B). In contrast, no symptoms were observed in tomato plants agroinoculated with pBin-SbYLCV-2A. When PCR was used to detect SbYLCV, negative results were found in the systemic leaves of agroinoculated tomato plants (Table 2). These results collectively suggest that the infectious clone of SbYLCV is infectious in N. tabacum, N. tabacum cv. Samsun, and N. glutinosa plants, but not in tomato plants.

A

Mock

SbYLCV
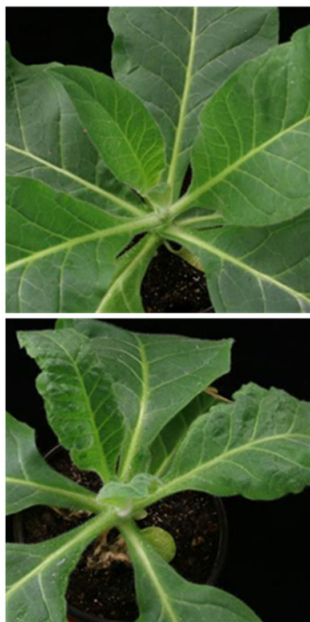

Nicotiana tabacum Nicotiana tabacum cv. Samsun NN cv. Samsun nn
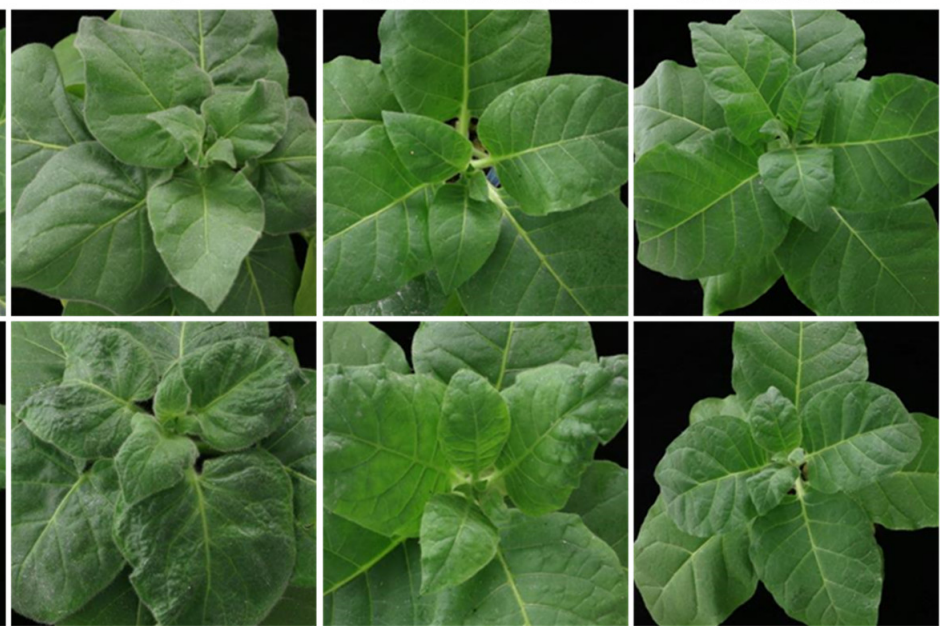

B

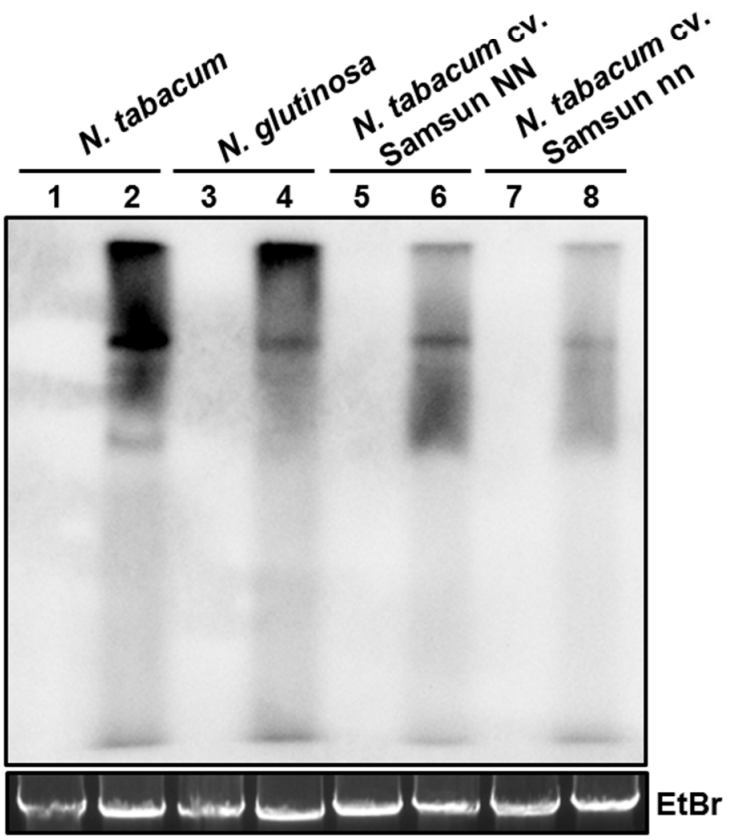

Figure 6. Systemic infection of SbYLCV in Nicotiana tabacum, N. glutinosa, N. tabacum cv. Samsun NN, and N. tabacum cv. Samsun nn plants. (A) Characteristic symptoms induced by SbYLCV. Plants were agroinoculated with the pBinPLUS vector (mock) or the infectious clone of SbYLCV, as indicated. Photos were taken at 30 days post-inoculation (dpi). (B) Southern blot hybridization analysis of SbYLCV DNA with a specific DIG-labelled DNA probe. Total DNA was extracted from systemic leaves of SbYLCV-infected and mock-inoculated plants, as indicated. Lanes 1, 3, 5, and 7 represent total DNA extracted from plants inoculated with the pBinPLUS vector; lanes 2, 4, 6, and 8 represent total DNA extracted from plants inoculated with the infectious clone of SbYLCV. Ethidium bromide staining of total DNA was used as a loading control. 


\section{Discussion}

Geminiviruses represent a threat to agriculture worldwide. In the last decade, viral metagenomic studies have contributed to the discovery of a growing number of geminiviruses. Here, we identified and characterized a novel monopartite geminivirus infecting soybean in China.

The arrangement of the six predicted ORFs encoded by the novel circular DNA virus is similar to that of monopartite begomoviruses, topocuviruses, turncurtoviruses, maldoviruses, and opunviruses. It also shares several common features with known geminiviruses, including the stem-loop structure and the conserved nanonucleotide, TAATATTAC, within the intergenic region and several critical motifs in the Rep ORF. However, pairwise sequence identity comparison and phylogenetic analyses of the full-length genome showed that this novel DNA virus is distinct from the established 14 genera of the Geminiviridae family, which only shares the highest sequence similarity $(65.6 \%)$ with ToLCJV. These observations suggest that this novel circular DNA virus represents a highly divergent species of a putative new genus of the Geminiviridae family, for which the name soybean yellow leaf curl virus (SbYLCV) is proposed.

Sequence analysis of the predicted SbYLCV proteins revealed that the Rep and C4 ORFs are closely related to those of begomoviruses, whereas the predicted V1, V2, C2, and C3 proteins share less than $52 \%$ amino acid sequence identity with those of other reported geminiviruses. This indicates that SbYLCV is a recombination-prone virus, which was further supported by six recombination detection methods implemented in the RDP software. Recombination is an important force driving geminivirus evolution, which can provide a strong selective advantage in virus species at the species and genus level. Previously, numerous begomoviruses have been reported to arise from recombination and the resulting recombinants often have an increased fitness or pathogenesis. The acquisition of a more virulent $\mathrm{C} 4$ arisen by recombination results in a more virulent begomovirus that does not require a betasatellite for symptom induction [27]. It would be interesting to understand whether novel roles have likely been acquired by SbYLCV during recombination.

Betasatellites are small circle ssDNA molecules that are frequently found in association with many begomoviruses and occasionally with mastreviruses [1]. In line with the deep sequencing data, no DNA B component, alphasatellite, or betasatellite was detected in the collected soybean sample by PCR. Infectivity tests with the constructed infectious clone further revealed the successful infection of SbYLCV in both the natural host soybean and the selected experimental hosts, conclusively demonstrating that SbYLCV is a monopartite geminivirus. Previously, betasatellites were proved to be replicated promiscuously by distinct geminiviruses and could be modified as a valuable versatile vector to suppress gene expression in plants [28-30]. It would be interesting to test whether the SbYLCV identified in this study could support the replication of different satellites, which would provide a valuable tool to study gene function in soybean plants.

Previous studies have shown that some functions of positional homologs such as Rep are conserved across geminivirus species and genera, whereas other geminivirusencoded proteins may display a diversity of functions in different species [31]. For example, several independent functions have been ascribed to C4/AC4 and C2/AC2 of different geminivirus species [32,33]. Recent years have witnessed an unprecedented understanding of the begomovirus protein structure and function; however, very limited information is available for the newly identified geminivirus species. Although SbYLCV has a similar genome organization to monopartite begomoviruses, topocuviruses, turncurtoviruses, maldoviruses, and opunviruses, the function of SbYLCV-encoded proteins remains to be explored. It is noteworthy that we applied the $10 \mathrm{kDa}$ threshold in the prediction of SbYLCV-encoded proteins, as shown in previous studies. A recent study showed that geminiviruses also encode small additional ORFs (size range 5-10 kDa) with specific subcellular localization and functions [34]. Additional ORF was also identified in the viral sense of the betasatellite genome and was demonstrated to have a critical role in virus 
infection [35]. These findings demand an exploration of small proteins in SbYLCV and an understanding of their roles in the infection cycle of SbYLCV.

Insect vectors play significant roles in geminivirus spread and evolution in nature, and $\mathrm{CP}$ is the only geminiviral protein that is involved in geminivirus transmission. The specificity between CPs with their insect vectors reflects an evolutionary relationship between geminiviruses and their transmission vector. A phylogenetic analysis of the predicted amino acid sequence of the $\mathrm{CP}$ of SbYLCV with the $\mathrm{CP}$ of geminiviruses representing all the 14 genera of the family Geminiviridae revealed that SbYLCV forms a distinct clade, but was most closely related to the $\mathrm{CP}$ of mastreviruses and ECSV. Mastreviruses are known to be transmitted by leafhoppers. Although the insect vector responsible for SbYLCV transmission in the field is scanty at this stage, the availability of the infectious clone developed here and their successful infection on soybean and other experimental hosts allow us to address this issue in the near future.

In summary, we have detected and characterized a novel soybean-infecting monopartite geminivirus in China. This novel geminivirus can replicate and establish a successful infection in inoculated soybean plants and five experimental hosts. These findings provide useful tools for the understanding of plant and geminivirus interactions and the selection of resistant soybean cultivars, which will be beneficial to the development of management strategies for disease control.

Supplementary Materials: The following is available online at https:/ /www.mdpi.com/article/10.3 390/v14020341/s1, Table S1. Sequences of primers used in this study; Figure S1. Matrix plot showing the pairwise identity of the complete genome sequences of SbYLCV and representative geminiviruses using the Sequence Demarcation Tool (SDT) software; Figure S2. Matrix plot showing the pairwise identity of the amino acid sequences of SbYLCV CP and the CP of representative geminiviruses using the SDT software; Figure S3. Neighbor-joining phylogeny tree constructed based on the amino acid sequences of SbYLCV Rep and the Rep of the representative geminiviruses. The statistical significance of the branches was estimated with a bootstrap of 1000 replicates; Figure S4. Matrix plot showing the pairwise identity of the amino acid sequences of SbYLCV Rep and the Rep of representative geminiviruses.

Author Contributions: Conceptualization, X.Y. and X.Z.; methodology, M.D., Y.W., C.C., X.L. and R.F.; software, M.D., Y.W. and C.C.; validation, M.D., Y.W., X.L., C.C. and R.F.; formal analysis, M.D., Y.W., C.C., X.L. and R.F.; investigation, M.D., Y.W., C.C., X.L. and R.F.; resources, M.D. and X.Y.; data curation, M.D., Y.W., C.C. and R.F.; writing-original draft preparation, M.D. and X.Y.; writing-review and editing, X.Y. and X.Z.; visualization, X.Y. and X.Z.; supervision, X.Y. and X.Z.; project administration, X.Y. and X.Z.; funding acquisition, X.Y. and X.Z.. All authors have read and agreed to the published version of the manuscript.

Funding: This work was financially supported by the National Natural Science Foundation of China (31972245 and 31720103914).

Institutional Review Board Statement: Not applicable.

Informed Consent Statement: Not applicable.

Data Availability Statement: Not applicable.

Conflicts of Interest: The authors declare no conflict of interest. The funding sponsors had no role in the design of the study; in the collection, analyses, or interpretation of data; in the writing of the manuscript, orin the decision to publish the results.

\section{References}

1. Yang, X.; Guo, W.; Li, F.; Sunter, G.; Zhou, X. Geminivirus-associated betasatellites: Exploiting chinks in the antiviral arsenal of plants. Trends Plant Sci. 2019, 24, 519-529. [CrossRef] [PubMed]

2. $\quad$ Rojas, M.R.; Macedo, M.A.; Maliano, M.R.; Soto-Aguilar, M.; Souza, J.O.; Briddon, R.W.; Kenyon, L.; Rivera Bustamante, R.F.; Zerbini, F.M.; Adkins, S.; et al. World management of geminiviruses. Annu. Rev. Phytopathol. 2018, 56, 637-677. [CrossRef] [PubMed] 
3. Vaghi Medina, C.G.; Teppa, E.; Bornancini, V.A.; Flores, C.R.; Marino-Buslje, C.; Lopez Lambertini, P.M. Tomato apical leaf curl virus: A novel, monopartite geminivirus detected in tomatoes in Argentina. Front. Microbiol. 2017, 8, 2665. [CrossRef] [PubMed]

4. Ma, Y.; Navarro, B.; Zhang, Z.; Lu, M.; Zhou, X.; Chi, S.; Di Serio, F.; Li, S. Identification and molecular characterization of a novel monopartite geminivirus associated with mulberry mosaic dwarf disease. J. Gen. Virol. 2015, 96, 2421-2434. [CrossRef]

5. $\quad$ Liang, P.; Navarro, B.; Zhang, Z.; Wang, H.; Lu, M.; Xiao, H.; Wu, Q.; Zhou, X.; Di Serio, F.; Li, S. Identification and characterization of a novel geminivirus with a monopartite genome infecting apple trees. J. Gen. Virol. 2015, 96, 2411-2420. [CrossRef]

6. $\quad$ Fontenele, R.S.; Abreu, R.A.; Lamas, N.S.; Alves-Freitas, D.M.T.; Vidal, A.H.; Poppiel, R.R.; Melo, F.L.; Lacorte, C.; Martin, D.P.; Campos, M.A.; et al. Passion fruit chlorotic mottle virus: Molecular characterization of a new divergent geminivirus in Brazil. Viruses 2018, 10, 169. [CrossRef]

7. Zerbini, F.M.; Briddon, R.W.; Idris, A.; Martin, D.P.; Moriones, E.; Navas-Castillo, J.; Rivera-Bustamante, R.; Roumagnac, P.; Varsani, A.; ICTV Report, C. ICTV virus taxonomy profile: Geminiviridae. J. Gen. Virol. 2017, 98, 131-133. [CrossRef]

8. Fiallo-Olivé, E.; Lett, J.M.; Martin, D.P.; Roumagnac, P.; Varsani, A.; Zerbini, F.M.; Navas-Castillo, J. ICTV Virus Taxonomy Profile: Geminiviridae 2021. J. Gen. Virol. 2021, 102, 001696. [CrossRef]

9. Nawaz-ul-Rehman, M.S.; Fauquet, C.M. Evolution of geminiviruses and their satellites. FEBS Lett. 2009, 583, 1825-1832. [CrossRef]

10. Strange, R.N.; Scott, P.R. Plant disease: A threat to global food security. Annu. Rev. Phytopathol. 2005, 43, 83-116. [CrossRef]

11. Hill, J.H.; Whitham, S.A. Control of virus diseases in soybeans. Adv. Virus Res. 2014, 90, 355-390. [PubMed]

12. Yang, X.; Du, M.; Li, S.; Zhou, X. Coinfection of cotton plants with watermelon mosaic virus and a novel polerovirus in China. Viruses 2021, 13, 2210. [CrossRef] [PubMed]

13. Zerbino, D.R.; Birney, E. Velvet: Algorithms for de novo short read assembly using de Bruijn graphs. Genome Res. 2008, 18, 821-829. [CrossRef] [PubMed]

14. Murray, M.G.; Thompson, W.F. Rapid isolation of high molecular weight plant DNA. Nucleic Acids Res. 1980, 8, 4321-4326. [CrossRef]

15. Muhire, B.M.; Varsani, A.; Martin, D.P. SDT: A virus classification tool based on pairwise sequence alignment and identity calculation. PLoS ONE 2014, 9, e108277.

16. Tamura, K.; Dudley, J.; Nei, M.; Kumar, S. MEGA4: Molecular evolutionary genetics analysis (MEGA) software version 4.0. Mol. Biol. Evol. 2007, 24, 1596-1599. [CrossRef]

17. Martin, D.P.; Lemey, P.; Lott, M.; Moulton, V.; Posada, D.; Lefeuvre, P. RDP3: A flexible and fast computer program for analyzing recombination. Bioinformatics 2010, 26, 2462-2463. [CrossRef]

18. Ma, Z.; Zhang, H.; Ding, M.; Zhang, Z.; Yang, X.; Zhou, X. Molecular characterization and pathogenicity of an infectious cDNA clone of tomato brown rugose fruit virus. Phytopathol. Res. 2021, 3, 14. [CrossRef]

19. Yang, X.; Ren, Y.; Sun, S.; Wang, D.; Zhang, F.; Li, D.; Li, S.; Zhou, X. Identification of the potential virulence factors and RNA silencing suppressors of mulberry mosaic dwarf-associated geminivirus. Viruses 2018, 10, 472. [CrossRef]

20. Deng, D.; McGrath, P.F.; Robinson, D.J.; Harrison, B.D. Detection and differentiation of whitefly-transmitted geminiviruses in plants and vector insects by the polymerase chain-reaction with degenerate primers. Ann. Appl. Biol. 1994, 125, 327-336. [CrossRef]

21. Bull, S.E.; Briddon, R.W.; Markham, P.G. Universal primers for the PCR-mediated amplification of DNA 1: A satellite-like molecule associated with begomovirus-DNAß complexes. Mol. Biotechnol. 2003, 20, 83-86. [CrossRef]

22. Briddon, R.W.; Bull, S.E.; Mansoor, S.; Amin, I.; Markham, P.G. Universal primers for the PCR-mediated amplification of DNAß Mol. Biotechnol. 2002, 20, 315-318. [CrossRef]

23. Ilyina, T.V.; Koonin, E.V. Conserved sequence motifs in the initiator proteins for rolling circle DNA replication encoded by diverse replicons from eubacteria, eucaryotes, and archaebacteria. Nucleic Acids Res. 1992, 20, 3279-3285. [CrossRef] [PubMed]

24. Koonin, E.V.; Ilyina, T.V. Geminivirus replication proteins are related to prokaryotic plasmid rolling circle DNA replication initiator proteins. J. Gen. Virol. 1992, 73, 2763-2766. [CrossRef] [PubMed]

25. Nash, T.E.; Dallas, M.B.; Reyes, M.I.; Buhrman, G.K.; Ascencio-Ibanez, J.T.; Hanley-Bowdoin, L. Functional analysis of a novel motif conserved across geminivirus Rep proteins. J. Virol. 2011, 85, 1182-1192. [CrossRef] [PubMed]

26. Lefeuvre, P.; Moriones, E. Recombination as a motor of host switches and virus emergence: Geminiviruses as case studies. Curr. Opin. Virol. 2015, 10, 14-19. [CrossRef] [PubMed]

27. Xie, Y.; Zhao, L.; Jiao, X.; Jiang, T.; Gong, H.; Wang, B.; Briddon, R.W.; Zhou, X. A recombinant begomovirus resulting from exchange of the C4 gene. J. Gen. Virol. 2013, 94, 1896-1907. [CrossRef]

28. Tao, X.; Zhou, X. A modified viral satellite DNA that suppresses gene expression in plants. Plant J. Cell Mol. Biol. 2004, 38, 850-860. [CrossRef]

29. Xu, X.; Qian, Y.; Wang, Y.; Li, Z.; Zhou, X. Iterons homologous to helper geminiviruses are essential for efficient replication of betasatellites. J. Virol. 2019, 93, e01532-18. [CrossRef]

30. Zhou, X. Advances in understanding begomovirus satellites. Annu. Rev. Phytopathol. 2013, 51, 357-381. [CrossRef]

31. Luna, A.P.; Lozano-Duran, R. Geminivirus-encoded proteins: Not all positional homologs are made equal. Front. Microbiol. 2020, 11, 878. [CrossRef]

32. Zhou, X. Hijack to escape: A geminivirus seizes a host imprinted E3 ligase to escape epigenetic repression. Sci. China Life Sci. 2021, 64, 323-325. [CrossRef] [PubMed] 
33. Medina-Puche, L.; Orilio, A.F.; Zerbini, F.M.; Lozano-Duran, R. Small but mighty: Functional landscape of the versatile geminivirus-encoded C4 protein. PLoS Pathog. 2021, 17, e1009915. [CrossRef]

34. Gong, P.; Tan, H.; Zhao, S.; Li, H.; Liu, H.; Ma, Y.; Zhang, X.; Rong, J.; Fu, X.; Lozano-Duran, R.; et al. Geminiviruses encode additional small proteins with specific subcellular localizations and virulence function. Nat. Commun. 2021, 12, 4278. [CrossRef] [PubMed]

35. Hu, T.; Song, Y.; Wang, Y.; Zhou, X. Functional analysis of a novel BV1 gene identified in a geminivirus betasatellite. Sci. China Life Sci. 2020, 63, 688-696. [CrossRef] [PubMed] 